\title{
Finite size scaling analysis with linked cluster expansions *
}

\author{
H. Meyer-Ortmanns ${ }^{\dagger}$ and T. Reisz ${ }^{\ddagger}$ \\ Institute of Theoretical Physics \\ University of Heidelberg \\ Philosophenweg 16 \\ D-69120 Heidelberg, Germany
}

October 25, 2018

\begin{abstract}
Linked cluster expansions are generalized from an infinite to a finite volume on a $d$-dimensional hypercubic lattice. They are performed to 20 th order in the expansion parameter to investigate the phase structure of scalar $O(N)$ models for the cases of $N=1$ and $N=4$ in 3 dimensions. In particular we propose a new criterion to distinguish first from second order transitions via the volume dependence of response functions for couplings close to but not at the critical value. The criterion is applicable to Monte Carlo simulations as well. Here it is used to localize the tricritical line in a $\Phi^{4}+\Phi^{6}$ theory. We indicate further applications to the electroweak transition.
\end{abstract}

\section{LINKED CLUSTER EXPANSIONS IN THE INFINITE VOLUME}

Convergent expansions such as linked cluster, hopping parameter or high temperature expansions provide an analytic alternative to Monte Carlo simulations. Originally they have been developed in the infinite volume, meanwhile they have been extended to finite volumes as well [1]. In contrast to generic perturbation theory, hopping parameter expansions (HPEs) are convergent expansions about completely disordered lattice systems. The expansion parameter $\kappa$ is the coefficient of the (pair) interaction term. Since we calculate free energies and connected correlations in the hopping parameter expansion, we generate linked cluster expansions (LCEs).

\footnotetext{
*Talk presented by H. Meyer-Ortmanns

$\dagger$ e-mail address: ortmanns@thphys.uni-heidelberg.de

‡Heisenberg fellow, e-mail address: t.reisz@thphys.uni-heidelberg.de
} 
LCEs have been generalized to scalar field theories at finite temperature by [2]. Compared to the zero temperature results, the finite temperature induces a tiny shift in the critical temperature (hopping parameter). Thus one has to face a similar problem as the improvement of a bad signal to noise ratio in Monte Carlo simulations. Here the signal corresponds to the finite temperature effect and the noise to the "background" of temperature zero graphs. The price to describe the critical region, in particular critical exponents, with an accurracy of $\approx 1 \%$ is to go to the 18 th order in the expansion in $\kappa$. This accurracy has been achieved in [2]. Thus the question arises, why we are still interested in a generalization of LCEs to a finite volume. The reason is twofold.

- The first one is to identify 1st order transitions from series expansions in the high temperature phase. For example a measurement of a vanishing critical exponent $\nu \cdot \eta$ is compatible with a Gaussian fixed point, but not conclusive for the onset of a first order transition region at a tricritical point. (The latter interpretation actually applies to our measurements.)

- The second reason is to distinguish second order transitions associated with different universality classes. Ideally critical exponents should show a clear gap between plateaus of different universality classes. Practically the gap is smeared out because of the truncation of the series expansions at finite order. The truncation has a similar rounding effect on this gap as a finite volume on thermodynamic singularities.

\section{GENERALIZATION OF HPEs TO A FINITE VOLUME}

\subsection{Embedding factors on the lattice}

In HPEs the action is split into a sum of ultralocal parts $\stackrel{\circ}{S}$ and a next neighbour part $S_{n n}$ with next neighbour couplings $\propto \kappa$. A Taylor expansion in $\kappa$ of the partition function $Z$ about the ultralocal contribution to $Z$ finally leads to graphical expansions of n-point susceptibilities $\chi_{n}$

$$
\chi_{n}(\kappa)=\sum_{\mu \geq 0} a_{\mu}^{(n)} \kappa^{\mu}
$$

The coefficients $a_{\mu}^{(n)}$ are a sum of graphs each of which consists of a product of the inverse symmetry factor, an internal symmetry factor, a lattice embedding factor, and a polynomial of vertex contributions depending on the couplings involved in $\stackrel{\circ}{S}$. It is only the embedding factor that depends on the topology of the particular lattice, that will change in passing from an infinite to a finite volume. 


\subsection{Shift in the critical coupling}

From our series expansions we measure a shift in $\kappa_{c}$ from a fit according to

$$
\ln \left(\left|\kappa_{c}(L)-\kappa_{c}(\infty)\right|\right) \simeq \ln c-y_{T} \ln L
$$

Here $c$ is a constant, $y_{T}=1 / \nu$ for 2 nd order transitions and $y_{T} \propto d$ for 1 st order transitions. The critical couplings $\kappa_{c}(L)$ and $\kappa_{c}(\infty)$ are determined as the radius of convergence of the series expansions in a finite or infinite volume, respectively. Since an extrapolation of the scaling behaviour from the height and width of the critical region is not feasible within the series expansions, we have proposed the following monotony criterion.

\subsection{The monotony criterion}

Consider two volumes $V_{1}$ and $V_{2}$ such that $V_{1}<V_{2} \leq \infty, 0<\alpha<1, \sigma$ stands for a generic coupling, and $t=\left(\kappa-\kappa_{c}\right) / \kappa_{c}$ for the reduced "temperature". We define

$$
r\left(V_{1}, V_{2}\right):=1-\frac{\chi_{2}\left(\kappa=\alpha \cdot \kappa_{c}, V_{1}\right)}{\chi_{2}\left(\kappa=\alpha \cdot \kappa_{c}, V_{2}\right)},
$$

The monotony criterion says that

$$
r_{V_{1}, V_{2}}\left\{\begin{array}{l}
>0,2 \text { nd order } \\
<0,1 \text { st order } \\
=0, \text { tricritical point for } \partial r / \partial \sigma \neq 0 .
\end{array}\right.
$$

The different behaviour of $r_{V_{1}, V_{2}}$ comes from the singular part of $\chi_{2}$. The underlying observation is a different monotony behaviour of response functions $\chi$ for 1 st and 2 nd order transitions that is seen for $\chi \mathrm{s}$ with nonanalytic behaviour in the infinite volume limit. The $\chi_{\mathrm{s}}$ are increasing in volume in a certain neighbourhood of $T_{c}$ for 2 nd order transitions and decreasing for 1st order transitions. For a precise specification of the scaling region, i.e. the bounds that $V_{1}, V_{2},|t|$ have to satisfy, we refer to [1]. The difference reflects the $\delta$-function and power law singularities for 1 st or 2 nd order transitions,respectively, predicted at $T_{C}$ in the thermodynamic limit.Thus the actual behaviour of $\chi_{2}$ we have found in applications to scalar $O(N)$ models (cf. section 3 ) is neither a pecularity of the model nor an artifact of the series expansions.

- It should be noticed that the criterion is not restricted to series expansions, but also applicable to Monte Carlo calculations (since both involved volumes may be finite) if the regular contribution to $\chi$ is really negligible.

- It is neither restricted to order parameter susceptibilities as the notation suggests, but similarly applies to other response functions like the specific heat if they diverge in the thermodynamic limit. 


\subsection{The effective potential for $V<\infty$}

The effective potential is defined via the effective action $\Gamma$ evaluated for a constant background field $M\left(L^{d} \cdot V_{\text {eff }}:=-\Gamma(M)\right)$. It can be expressed in terms of the 1PI-susceptibilities according to

$$
\begin{gathered}
V_{e f f}(M)=\frac{1-4 D \kappa \chi_{2}^{1 P I}}{\chi_{2}^{1 P I}} \frac{M^{2}}{2}-\frac{\chi_{4}^{1 P I}}{\left(\chi_{2}^{1 P I}\right)^{4}} \frac{\left(M^{2}\right)^{2}}{4 !} \\
-\frac{1}{\left(\chi_{2}^{1 P I}\right)^{6}}\left(\chi_{6}^{1 P I}-\frac{10\left(\chi_{4}^{1 P I}\right)^{2}}{\chi_{2}^{1 P I}}\right) \frac{\left(M^{2}\right)^{3}}{6 !}+O\left(M^{8}\right) .
\end{gathered}
$$

The $\chi_{2 n}^{1 P I} \mathrm{~S}$ are obtained from the $\chi_{2 n} \mathrm{~S}$ if the graphical expansion of the $\chi_{2 n} \mathrm{~S}$ is restricted to 1PI graphs. The (non)convex shape of the effective potential in the symmetric phase indicates the order of the transition. Its evaluation in a finite volume leads to an alternative determination of $\kappa_{c}(L)$ via the coexistence of different minima of the free energy or a vanishing of the coefficient of the quadratic term.

\section{RESULTS FOR A $3 d \Phi^{4}+\Phi^{6}$ THEORY}

We apply the finite size scaling analysis with HPEs to a scalar theory with $\Phi^{4}$ and $\Phi^{6}$-terms and $O(N)$ symmetry in 3 dimensions, $N$ is chosen as 1 and 4 . After a suitable reparametrization the ultralocal part of the action reads

$$
\stackrel{\circ}{S}(\Phi, \lambda, \sigma)=\Phi^{2}+\lambda\left(\Phi^{2}-1\right)^{2}+\sigma\left(\Phi^{2}-1\right)^{3}
$$

The hopping parameter term is given as

$$
S_{n n}=-2 \kappa \sum_{x, y \mathrm{nn}} \Phi(x) \cdot \Phi(y) .
$$

For a suitable choice of couplings the model has a tricritical line separating regions of first and second order transitions. Thus it serves as an appropriate toy model for testing our methods.

A fit of the shift in $\kappa_{c}$ according to Eq. 2 leads to the values of $y_{T}$ listed in the Table.

\begin{tabular}{rrrr}
\hline$N$ & $y_{T}$ & & order \\
\hline 1 & $6.068(43)$ & $\approx 2 d$ & 1 st \\
4 & $5.55(59)$ & $\approx 2 d$ & 1 st \\
1 & $2.656(48)$ & $\approx 2 / \nu$ & 2 nd \\
4 & not conclusive & & 2 nd \\
\hline
\end{tabular}

The value for $y_{T}$ for the first order transitions confirms results of [3] for $N=1$ and extends results of [4] for $N=4$. It disagrees with the naive expectation of a scaling with $d$ rather than $2 d$. The figure shows results for the monotony 


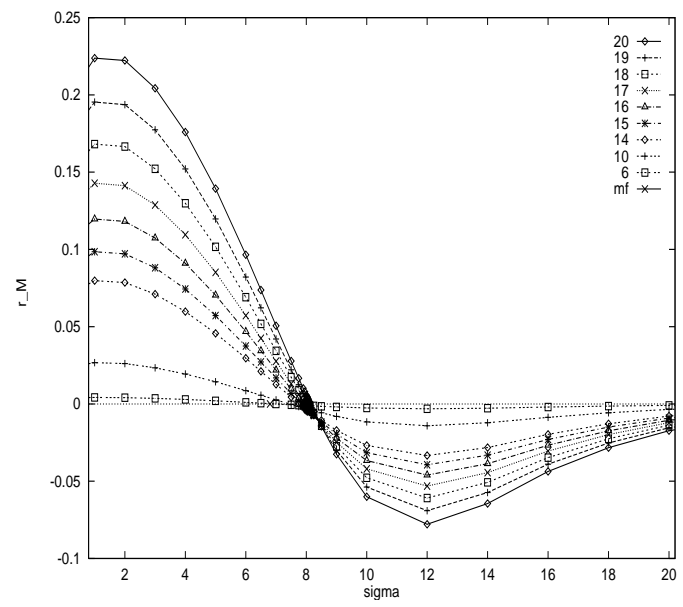

criterion applied to the special case of $V_{1}=4^{3}, V_{2}=\infty^{3}, \alpha=0.98, N=4$, $\lambda=\sigma / 2$. The additional index $M$ refers to the order of truncation. A similar figure is obtained for $r\left(V_{1}, V_{2}\right)$ as function of $\sigma$ for fixed order of the truncation and varying volumes. Thus the intersection of the curves with the $\sigma$-axis, which localizes the tricritical point, weakly depends on the order of the truncation and the choice of $V_{1}$. The ultimate determination of the tricritical couplings $\sigma_{t}$ and $\lambda_{t}$ needs an extrapolation in both parameters. Respecting the mutual relation between the truncation effect and the finite volume dependence of the intersection, the final estimate for $\sigma_{t}$ leads to $\sigma_{t}=9.454(49)$. For further details on the involved extrapolations we refer to [1]. This result improves the infinite volume estimate of $8 \leq \sigma_{t} \leq 10$ by two orders of magnitude. It should be noticed that the volume independence of $\chi_{2}$ along the tricritical line supports the validity of a mean field analysis of tricritical exponents even in three dimensions as one might have expected from the Ginzburg criterion. The localization of $\sigma_{t}$ from the change in the shape of the effective potential leads to an upper bound on $\sigma_{t}$, we find $9.75 \leq \sigma_{t} \leq 10.0$, since the involved systematic errors are less well under control.

An extension to effective scalar models for an underlying Salam-Weinberg theory with HPEs in a finite volume will particularly well work in the range of large Higgs masses. It complements the range of Higgs masses which has been available in recent Monte Carlo simulations and allows a determination of the critical Higgs mass above which the electroweak transition turns into a smooth crossover phenomenon [5].

\section{References}

[1] H. Meyer-Ortmanns and T. Reisz, Critical Phenomena with Linked Cluster Expansions in a Finite Volume heplat/9604006.

[2] T. Reisz, Nucl. Phys. B450 (1995) 569; Phys. Lett. B360 (1995) 77. 
[3] C. Borgs, R. Kotecky, Phys. Rev. Lett. 68 (1992) 1734.

[4] C. Borgs, R. Kotecky, S. Miracle-Sole, J. Stat. Phys. 62 (1991) 529.

[5] H. Meyer-Ortmanns, I.-O. Stamatescu, T. Reisz, and T. Takaishi, work in progress. 